\title{
Physical, optical, and electrical properties of a new conducting polymer.
}

\begin{abstract}
This work studies the electrical and optical properties of the conducting polymer composite films of polypyrrole-chitosan (PPy-CHI). The surface plasmon resonance (SPR) technique was used to study the optical properties of PPy and PPy-CHI composite films. Then, the values of the real and imaginary parts of the refractive indexes of PPy and PPy-CHI films were obtained by nonlinear least square fitting using Fresnel equations for a three-layer system of SPR system. The electrical conductivity measurements showed that the conductivity of the electrochemical prepared films improved in the presence of CHI and can be controlled by varying the CHI amount in the composite. The thermal diffusivity of the PPy-CHI composite films was measured by open photoacoustic spectroscopy and it has been shown that the thermal diffusivity is related to the electron migration in the conjugation chain length. The increase in electromagnetic interference shielding effectiveness (EMI SE) with the increase in electrical conductivity of the films is mostly from shielding by reflection rather than absorption.
\end{abstract}

Keyword: Electrical properties; Polymer composite films; Electrochemical. 